\title{
Biomechanics of Kumite Style Gyaku tsuki in Karate
}

\author{
Jay Venkatraman and Mohammadreza Nasiriavanaki* \\ Department of Biomedical Engineering, USA \\ *Corresponding author: Nasiriavanaki M, Department of Biomedical Engineering, USA
}

\begin{tabular}{|c|c|}
\hline ARTICLE INFO & ABSTRACT \\
\hline Received: February 02, 2019 & \multirow{3}{*}{$\begin{array}{l}\text { In karate sparring (Kumite), punches are used more than kicks to score points. Among } \\
\text { these punches, the gyaku tsuki is the most commonly used punch. The objective of the } \\
\text { punch is to hit the target at a medium range in a very short time producing maximum } \\
\text { force. In this study we propose to design a new standalone system to measure the speed } \\
\text { and force generated by the punch. Finite Element Analysis (FEA) has been performed to } \\
\text { determine why the specific sensor arrangement works efficiently, distributing the force } \\
\text { on all sensors. Since punches are executed within a few hundred milliseconds, identifying } \\
\text { the punch which is faster by even a few milliseconds would give an edge in competitions. } \\
\text { Two types of gyaku tsuki in Kumite stance were compared for speed and force generated-a } \\
\text { normal gyaku tsuki and a gyaku tsuki without rotating the fist. }\end{array}$} \\
\hline Published: February 12, 2019 & \\
\hline $\begin{array}{l}\text { Citation: Jay Venkatraman, Moham- } \\
\text { madreza Nasiriavanaki. Biomechanics } \\
\text { of Kumite Style Gyaku tsuki in Karate. } \\
\text { Biomed J Sci \& Tech Res 14(3)-2019. } \\
\text { BJSTR. MS.ID.002550. }\end{array}$ & \\
\hline
\end{tabular}

Keywords: Gyaku tsuki; Punch; Karate; Martial Arts; Force, Speed

\section{Introduction}

Karate training is divided in three main parts- kata (forms), kihon (basics) and Kumite (sparring). The strikes in karate are mostly linear [1] i.e. they travel in a straight line which makes them quick. Mass of the leg is about $20 \%$ while that of the arm is just $5 \%$ of the total body weight [2]. This difference in mass is why kicks harder to aim but are more powerful. In competitions, scoring precise points quickly is emphasized than delivering powerful blows. Hence, punches find a very important place in the karate Kumite arsenal. Gyaku tsuki also known as the reverse punch is the most frequently used punch in competitions. The objective of the punch is to hit the target at a medium range in a very short time producing maximum force. From normal fight stance, the attacker athlete lowers his centre of mass while extending his stance lunging towards his opponent and extending his punching arm forward executing the punch in the open unguarded part of the opponent's abdomen [3]. This punch is preceded by a series of body movements namely the twisting of hips, shoulder rotation. The peak velocity of the joint preceding a joint, aids in further increasing the joint's peak velocity. Rathee et al. [4] conducted a study for the parameters required for breaking a wooden board by two karatekas. For inelastic collision, Deformation Energy (DE) is the energy lost to deformation. It is given by

$$
\text { D.E. }=(1 / 2)\left(\left(m_{1} \times m_{2}\right) /\left(m_{1}+m_{2}\right)\right) \times V_{c}^{2}
$$

where $m 1$ is the mass of the wooden board and $m 2$ is the mass of the arm of the punching participant. $V_{c}$ is the velocity of the punch upon impact. In the above equation, producing and hitting the target with maximum energy is the aim. Mass of the arm and velocity are the two parameters which govern the generation of this energy. Mass of the arm would remain the constant or would not bring about any significant change even if trained hard. Hence, karatekas should train to increase their punch velocity. Increase in punch velocity twice would quadruple the energy production. Punching is studied as a kinetic link principle where the joints' movement occur in a sequential order. The peak velocity of the next distal joint exceeds the peak velocity of the preceding proximal joint [5]. Since the pelvis and trunk of the body have more mass these body parts while rotation have greater moment of inertia. During movement, the (relatively) larger muscles attached to these joints contract and achieve their peak angular velocity earlier than the next segment.

After reaching their peak velocities, these muscles relax transferring this velocity to the next distal segment [6]. Dyson et al. [7] did a study of the muscles involved in a boxing punch to 
understand the role of major individual muscles in the punch. It was found out that the gastrocnemius was the first muscle to be activated during a punch due to the action of moving the body forward by plantarflexion. The rectus femoris and biceps femoris were activated next to extend the knee and hips. These were followed by the trapezius, deltoid muscles and biceps brachii to flex the elbow shortly, followed by extension of the elbow done by the triceps brachii and flexor carpi radialis in the forearm to execute the punch. Over the past few decades, different methods have been used to try and calculate the absolute force of a martial artist's punch and kicks for research purposes. Researchers at Poland have developed a punching bag with embedded strain gauge to measure force of a punch [8]. They improved it by using embedded accelerometers and a larger bag which would calculate forces acting on the bag and separate the force components in the three direction axes.

Another study done by Estevan et al. [9] uses motion capture and force plates to calculate the ground reaction force on the legs while striking and targets placed on specific joints on the limb aided in determining the kinematics of the arm. Another system built based on the target lever arm's torsional movement along the target stand gave force values acting on the target [10]. Studies conducted by Estevan et al. and Falco et al. [11-14] used piezoresistive sensors to measure force acting on the sensors. The system designed in this study is based off this primary design. A thesis study done by Singh Anupam uses a force measuring instrument FGV SHIMPO to measure force. Five springs were added behind a stiff punching board to dampen the force values and a study with the relative force values was conducted. For his specific study, calculating absolute force values were not necessary [2]. Girodet et al. [15] designed and used an instrumented makiwara to measure the force of the reverse punch.

\section{Materials and Methods}

The method used by Falco et al. [14] to measure the force of kicks in taekwondo was adapted and modified into a standalone system which was able to detect the speed and force of a gyaku tsuki punch while displaying it in real time. Two wooden circular plates of wood were cut in a dimeter of $25 \mathrm{~cm}$ and sensors were placed between them to detect force applied on the wooden platform. The force is distributed homogenously throughout the wooden surface and recorded by the sensors. The sensors used were Tekscan Flexiforce A201 force sensors [16]. Nine sensors were arranged along the sides of an equilateral triangle of length $15 \mathrm{~cm}$. Three sensors were arranged along the midpoint of each side of the triangle as shows in Figure 1. The sensors were placed in between two spacers on each side such that when the wooden plates undergo compression, they do not touch each other dissipating the force amongst themselves since the sensor is only $0.2 \mathrm{~mm}$ thin. This arrangement was decided by selecting the best among 15 simulated designs (designs a-o) using Finite Element Analysis techniques in Hypermesh.

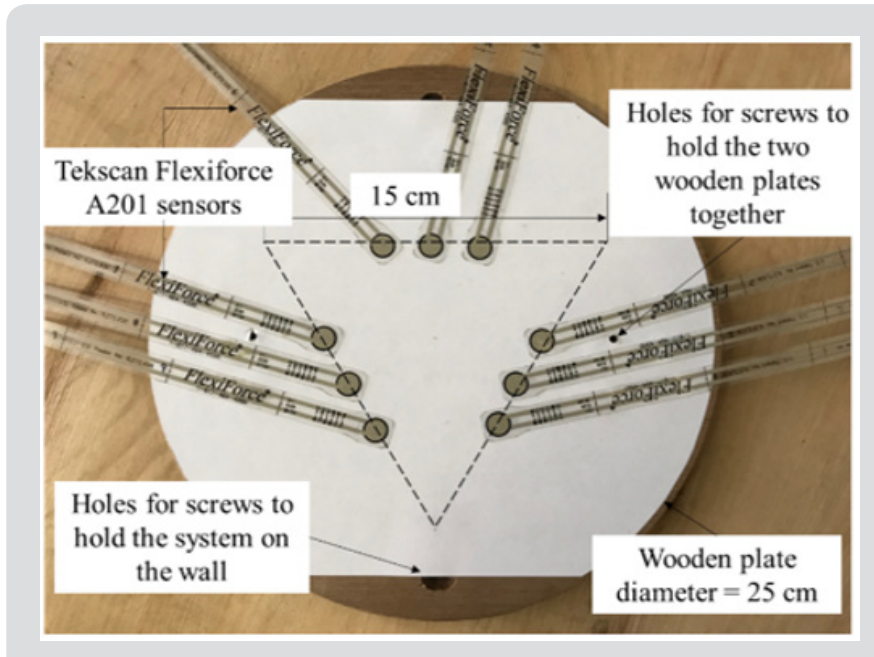

Figure 1: The nine A201 flexi force sensor arranged along the sides of an equilateral triangle of length $15 \mathrm{~cm}$.

To simulate the model in Hypermesh, the outline of the model was first designed in SolidWorks and imported in Optistruct. Wood and sensor components were created, material properties were assigned, and constraints were defined. The components were meshed using $5 \mathrm{~mm}$ and $1 \mathrm{~mm}$ mesh size respectively. A distributed load of total $2 \mathrm{kN}$ was added at every node inside a rectangular area of side length $80 \times 60 \mathrm{~mm}$ considering the size of the fist. The model was run and total von misses stress on all elements of the sensors were checked. Studies by Krzystof Busko et al. [17-19] states that the maximum force delivered by a boxer is about 4.5 to $5 \mathrm{kN}$. Studies by Karpilowski $[20,21]$ state that a boxer's maximum striking force 2697N. Different studies indicate different values depending upon the methodology used (Figure 2). Since this study makes use of 9 sensors having force range of $455 \mathrm{~N}$ each, there was no need to further increase the sensing range of the sensor. The wood thickness was chosen as 0.5 inch such that the wooded plate can withstand higher forces up to $5 \mathrm{kN}$ without any permanent deformation.

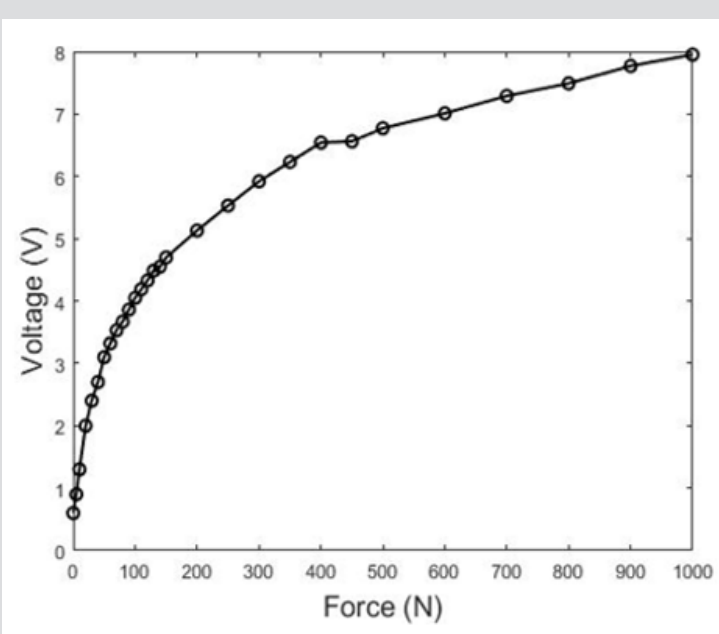

Figure 2: Calibration curve obtained by the Instron machine loading force on the system vs voltage obtained. 
Four holes were made through the two wooden plates- two for holding the wooden plates together and two for hanging the system to the wall. A foam of $2.54 \mathrm{~cm}$ thickness and $20 \mathrm{~cm}$ diameter was placed on the punching surface to protect the user's hands from injuries. After securing the wooden plates together with screws such that they do not move with respect to each other laterally and disturb the sensor arrangement, the entire system was calibrated under the instron machine by adding loads up to $1000 \mathrm{~N}$ and corresponding voltage values were noted. In the force calculation algorithm, these values were interpolated to obtain the force values.

\section{Punch Speed Detection Module}

To detect the punch speed, two lasers (KY-008 red dot diode laser)-sensor pairs were placed on a wooden stand $10 \mathrm{~cm}$ from each other with the laser beam's direction perpendicular to the direction of punch speed. The sensors have a hollow cylindrical covering around them to avoid ambient light disturb the sensor signals. When the punching hand interrupted these signals, time $t_{1}$ and $t_{2}$ were noted down. $t_{3}=t_{2}-t_{1}$ was the time in which the punch travelled the distance of $10 \mathrm{~cm}$. Speed of the punch was calculated,

$$
\text { speed }=100 / t_{3} .
$$

\section{Components Used}

A SainSmart 3.2" TFT LCD was used for displaying the values. The LCD was connected to the Arduino Mega board using an Arduino mega shield for the LCD. On/off and reset buttons were soldered to the circuit and set up on the surface of the circuit box (Figures 3 \& 4).

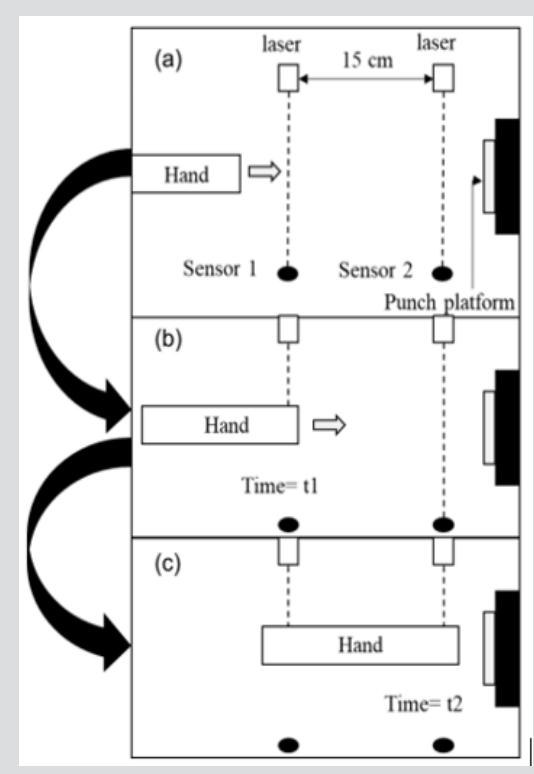

Figure 3: Speed detection module working principle. (a) setup initial position, (b) hand interrupts signal to sensor 1- time $t_{1}$ is noted, (c) hand interrupts signal to sensor 2time $t_{2}$ is noted.

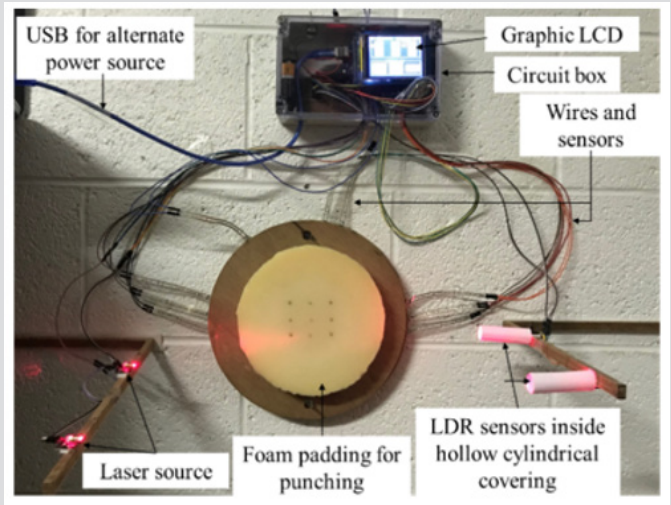

Figure 4: Arrangement of the system on the wall.

\section{System Specifications}

Using the calibration curve values, the specifications of the system were determined. The calibration curve follows a logarithmic pattern. The sensitivity of the system is 0.015 volts/ newton upto $400 \mathrm{~N}$ and it decreases to 0.0024 volts/newton upto $1000 \mathrm{~N}$. The system has a resolution of 79.5 volts. Due to the weight of the wood, the minimum value obtained is $0.6 \mathrm{~V}$ and upon application of $1000 \mathrm{~N}$, on top of the wooden platform, the voltage obtained is $7.95 \mathrm{~V}$.

\section{Test Conducted}

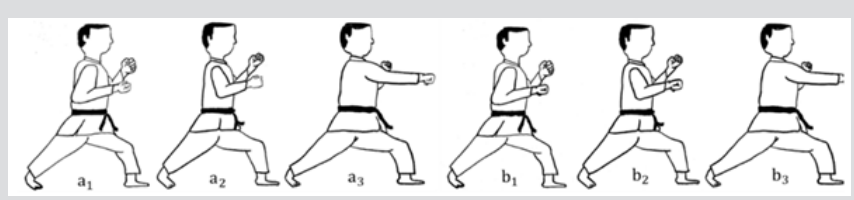

Figure 5: (a1) starting, (a2) mid and (a3) final position of normal gyaku tsuki; (b1) starting, (b2) mid and (b3) final position of gyaku tsuki without fist rotation.

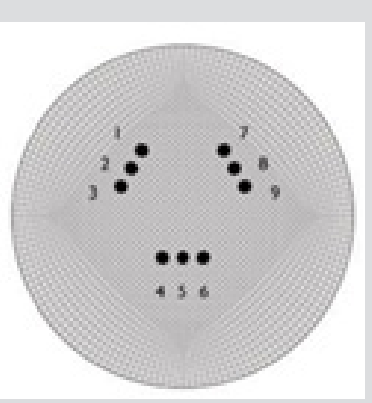

Figure 6: Sensor arrangement in between wooden plates.

One participant was chosen for this test- weight $72 \mathrm{kgs}$, about 7 years of experience in karate. The participant was asked to punch the system with normal gyaku tsuki and gyaku tsuki without rotating the fist both in Kumite style as shown in Figure 5. All these punches were done from a stationary position without any stepping motion. The forces and speed of these punches was to be determined to analyse the best type of gyaku tsuki to be used in competitions. The 
Wayne State University IRB cleared the participant for the tests. The participant was asked to stand at a comfortable arm's length for punching the system and to maintain the Kumite stance to maintain balance since punching on the rigid wall tended to throw the participant off balance. Figures 5 \& 6 shows the initial, middle and final position of the fist while punching the two punches. For punching through various speeds from 1 to $6 \mathrm{~m} / \mathrm{s}$, different force values were calculated and plotted. While punching the participant was instructed not to be bias towards any specific punch type. The participant was also instructed not to compress the wood as the algorithm would not accept that as a valid reading. Any value for punch which would have a plateau at the peak value would be rejected.

\section{Results}

Falco et al. [14] had initially designed this system to measure forces of kicks of taekwondo practitioners. He had arranged 9 the sensors in the form of an equilateral triangle of length $15 \mathrm{~cm}$ keeping 3 sensors near the midpoint on all the three sides of the triangle. It wasn't clear why this specific design was chosen by the author since essentially covering the punching area by even 3 or 4 sensors around it should be able to record the loading like working of strain gauges in a weighing scale. Finite Element Analysis (FEA) of the wood and sensor arrangement was done simulating different punching regions. Hypermesh software was used for this FEA. The white outer circle is the wood and small black circles are the sensors. The force on sensors displayed in Figure 7 follow the numbering shown in Figure 6. The figures below show the several regions where the punch was simulated, and the corresponding sensor forces recorded. Four different regions were simulated to check the response- the center where the force on sensors is most distributed, inside the area formed by the sensors but away from the center, halfway outside the triangular area on the sensors and completely outside the area.

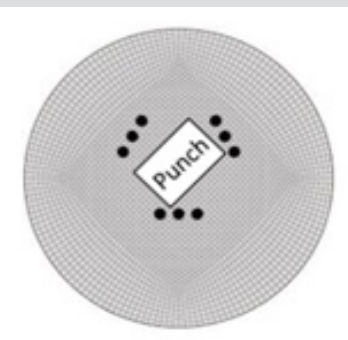

Punch at center

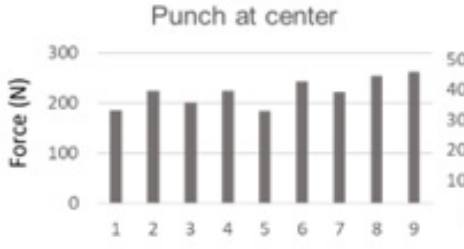

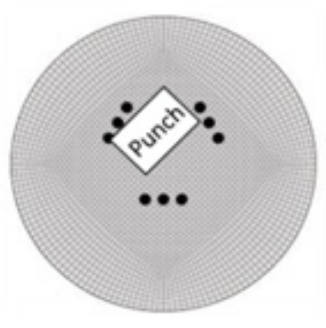

Punch inside area

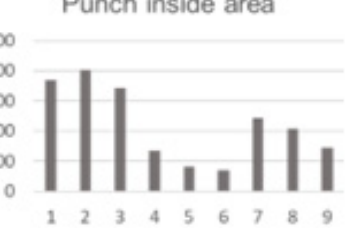

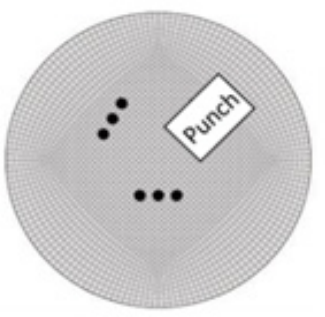

Punch on sensors

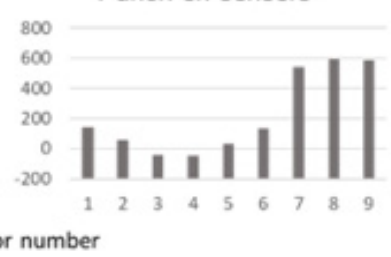

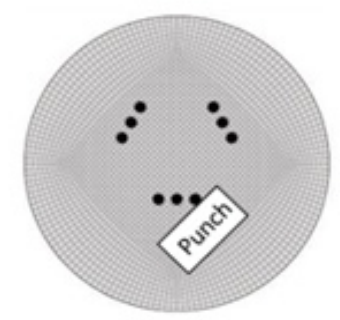

Punch away from sensors

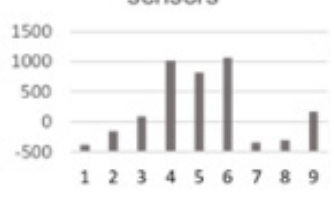

Figure 7: Sensor force readings when punched on different areas on the wooden plates.

Tests were conducted to check the experimental values when punched with both the types of punches. Figure 8 shows the curve of force vs speed of both the punches while Table 1 shows these values. After checking the force trend generated by these punches, both the punches were tested for achieving maximum average speed. Table 2 shows the tests conducted to calculate the maximum average speed that can be attained by both these punches.

Table 1: Speed and corresponding force values of the normal gyaku tsuki and the gyaku tsuki without fist rotation.

\begin{tabular}{|c|c|c|c|}
\hline \multicolumn{2}{|c|}{ Normal gyaku tsuki } & \multicolumn{2}{c|}{ Gyaku tsuki without rotation } \\
\hline Speed (m/s) & Force (N) & Speed (m/s) & Force (N) \\
\hline 1.92 & 142 & 1.69 & 147 \\
\hline 2.27 & 180 & 1.89 & 170 \\
\hline 2.78 & 284 & 2.86 & 266 \\
\hline 3.23 & 320 & 2.94 & 291 \\
\hline 3.85 & 349 & 3.57 & 317 \\
\hline 4 & 366 & 4.17 & 320 \\
\hline
\end{tabular}

\begin{tabular}{|l|l|l|l|}
\hline 4.55 & 378 & 4.35 & 331 \\
\hline 5.26 & 384 & 4.55 & 337 \\
\hline
\end{tabular}

Table 2: Maximum attainable speeds $(\mathrm{m} / \mathrm{s})$ of both the punches.

\begin{tabular}{|c|c|c|c|}
\hline Test no. & Normal gyaku tsuki & $\begin{array}{c}\text { Gyaku tsuki without fist } \\
\text { rotation }\end{array}$ & Force (N) \\
\hline 1 & 5 & 5.26 & 147 \\
\hline 2 & 5.56 & 5.56 & 170 \\
\hline 3 & 5.26 & 5.65 & 266 \\
\hline 4 & 5.26 & 5.88 & 291 \\
\hline 5 & 5.56 & 5.26 & \\
\hline Average & 5.33 & 5.52 & \\
\hline
\end{tabular}

\section{Discussion}

\section{Sensor Arrangement}

The sensor arrangement adopted by Falco et al. [14] was used and simulated to verify how forces are being distributed on all the sensors when punched in different areas of the wooden punching 
platform. Ideally a load would need to be supported by a base on at least three sides. Placing a load inside the area enclosed by the sensors would distribute all the load on them whereas if the load is placed outside the area enclosed by the sensors, not all of the force would be recorded by the sensors. Moreover, if all sensors read forces equally, then the effective range required by each sensor is reduced and it would be more effective rather than forces being distributed in an uneven pattern when punched near one sensor and away from other.

As seen from (Figure 7), sensors record force more evenly when punched at the centre than away from the centre. In case 2 , there is a big gap between the highest and lowest readings on the sensors since it is closer to a certain group of sensors than the other. In case 3, the gap is higher due to the introduction of negative forces. Since the load extends in the area not supported by the sensors, an imbalance is created and the sensors on the other end display a negative force. The negative force acts by pushing the board away from the sensor. The resultant of all these forces is $2 \mathrm{kN}$. In case 4 , negative forces are higher because the board is tilting a considerable distance on one side.

Flexiforce A201 sensor can record only $445 \mathrm{~N}$ without increasing its sensing range. In case 1 , all sensors are able to record values since the values are well within range. In case 2 , the highest force is $405 \mathrm{~N}$ which means this case is almost on the verge of its range for recording force. Hence, punching any further on the sensors would saturate the readings on the sensors. The highest readings in case 3 and 4 are $591 \mathrm{~N}$ and $1070 \mathrm{~N}$ respectively which is out of the sensors sensing range. Hence this specific arrangement works for recording punch forces fully inside the triangular area.

\section{Comparison of Punch Force}

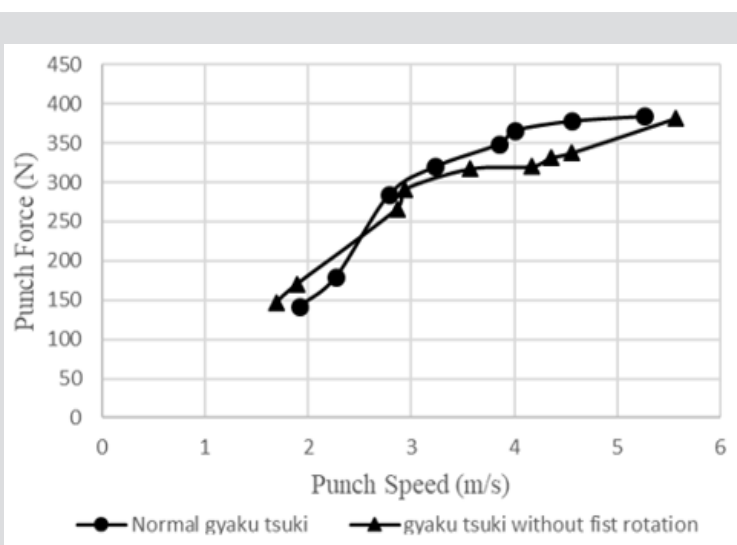

Figure 8: Speed v/s force of both punches.

The values obtained from programmable microcontroller displays force values in Newtons on the graphic LCD along with the force time curve thus providing real time data to the user. The values obtained in Table 1 and Figure 8 show that the force generated by the normal gyaku tsuki is higher than its counterpart when punched at the same speed. At all speeds the normal gyaku tsuki's force is $6.67 \%$ to $12.16 \%$ higher than the gyaku tsuki without fist rotation. Diacu, [22] showed the mathematical proofs for increase in generation of Kinetic Energy when performing a normal gyaku tsuki. Considering a $70 \mathrm{Kg}$ person punching at a speed of $5.5 \mathrm{~m} / \mathrm{s}$, the mass of his arm would be $5 \%$ of his total weight. He rotates his fist by 180 degrees or $5 \pi$ radians and the radius of his fist rotation be $5 \mathrm{~cm}$ or $0.05 \mathrm{~m}$. During translational punching, the generated kinetic energy, Ek and rotational Energy (Er) were calculated to be 52.94J and $1.079 \mathrm{~J}$ respectively.

This proves that the force generated by the gyaku tsuki is higher than the gyaku tsuki without rotation. Research on the change in moment arm during pronation and supination has been done by Bremer, [23]. Consider the 0 position of the wrist to be the position where the line joining the radial and ulnar styloids are parallel to the axis of the humerus with the elbow flexed 90 degrees. The muscles in the forearm responsible for the pronation of the forearm are pronator teres, pronator qudratus, flexor carpi radialis, palmaris longus and extensor carpi radialis longus. The change in moment arm is higher during pronation of the arm than during supination. The maximum moment arm values are seen when the pronation and supination are about 40 degrees. Due to the increase in moment arm, there is change in moment when the arm is rotated while punching the normal gyaku tsuki. This increase in moment is the possible reason for the higher force generation.

\section{Comparison of Punch Speed}

Maximum attainable speeds of both the punches were checked in Table 2 to determine whether either punch can punch with higher speed than the other. It was seen that the gyaku tsuki without the fist rotation can attain final speed of 5.52 (S.D. 0.212) m/s while the normal gyaku tsuki could attain an average high speed of 5.33 (S.D. 0.215)m/s. This $3.56 \%$ difference in speeds show that both the punches attain almost the same average high speeds.

\section{Conclusion}

The specific design was chosen because it was used by Coral Falco et. al. for their study to find out the force of a taekwondo kick. Their system has been modified by adding a speed detection module and a graphic LCD which displays the punch speed, force, force time curve of the punch type in real time. This system can be used to determine all these parameters for any type of punch except for any punch which has a pushing movement. Any movement aiding the punch like the yori Yashi (forward step)- gyaku tsuki/ kizmi tsuki can be used too. The system has a relatively low sampling rate hence punch speeds upto only $8.33 \mathrm{~m} / \mathrm{s}$ are recorded. The curves obtained from the punches prove that with increase in punch speed, the force of the punch increases. By simulating the force distribution on all sensors by punching in different areas of the wooden platform, it was seen that the sensors record force when the whole punch lands inside the area of the triangle formed by the sensors. As per the results of speed and force obtained by the two punches obtained in Table 1 and the pattern observed in Figure 8, the normal gyaku tsuki delivers a higher force upon impact when punched than the gyaku tsuki without fist rotation. 
The group of muscles involved in pronation motion of the wrist and forearm while performing the normal gyaku tsuki generated a higher moment which leads to higher force generation. It is in accordance with its speed being slightly higher than the latter punch. There is a variable difference in force about $6.67 \%$ to $12.16 \%$ at variable speeds. The difference in speeds are due to human error as there may have been some inconsistencies while punching. The experiments to determine the average maximum attainable speed also revealed that both the punches punch with an approximate same speed. In a kumite match, the gyaku tsuki without rotation should be used since punching in this manner produces slightly higher speed which is the key in all styles in karate. Force isn't emphasised except in hard contact styles like kyokushin. For semi or light contact styles like Shotokan and according to WKF (world Karate Federation) rules, the gyaku tsuki without rotation should be used.

There are some techniques that could be followed to increase the speed of any punch. Rotating the pelvis transfers the increase in angular velocity to the arm. Studies done previously have proved that gyaku tsuki punched from near the waist rather than starting midway produces more linear velocity of the wrist [24]. Also, faster the opposite arm is retracted, faster is the punch [6,25]. There have been other studies stating different force values of punches and kicks in the past. The absolute force value of the punch is not obtained in this system since calibration of the system was done by placing static loads whereas the punch is a dynamic load. Moreover, since the sampling rate is less at $134 \mathrm{~Hz}$, it is not able to record any higher peaks which could have been missed. Using this very system repeatedly would give the results in real time and the user can mark one's progress through practice and set short and long-term goals. Determining the kinematics of the arm would provide side by side comparison of the arm velocity changes w.r.t time and more conclusions could be drawn from that. EMG can be recorded of the muscle groups to find the function of individual muscle and groups in a punch. More participants should be tested to strengthen the claim.

\section{Acknowledgment}

We are thankful to my friends who taught me to do simulations using Hypermesh and the Wayne State University Machine Shop for letting me borrow their tools, some miscellaneous materials required and helping me getting my system ready.

\section{References}

1. Arus E (2012) Biomechanics of human motion: Applications in the martial arts. CRC Press, pp. 559.

2. Anupam S (2017) Analysis of force, time, energy, psychological demand and safety of common kicks in martial arts. Industrial Engineering, Iowa State University, Ames, p. 1-46.

3. Ciubucciu Ionete G, Mereuta E Biomechanics of karate techniques. "Dunarea de Jos" University of Galati, Romania.

4. Rathee N, Magnes J, Davis J (2014) Kinematics of board breaking in karate using video analysis-a dynamic model of applied physics and human performance. European Scientific Journal 10(12).
5. Ionete GL, Mereuta E, Mereuta C, Tudoran MS, Ganea D (2011) Linear kinematic analysis of gyaku-tsuki karate technique. The Annals of "Dunarea De Jos" University of Galati 2011: 1.

6. Gallaher DM (2013) 3D analysis of punching technique: Reverse vs. lead (Gyaku tsuki vs. Oi tsuki). p. 1-70.

7. Rosemary Dyson, Marcus Smith, Christopher Martin, Lisa Fenn (2007) Muscular recruitment during rear hand punches delivered at maximal force and speed by amateur boxers. ISBS-Conference Proceedings Archive.

8. Krzysztof Buśko, Zbigniew Staniak, Patrycja Łach, Joanna Mazur Różycka, Radosław Michalski, et al. (2014) Comparison of two boxing training simulators. Biomedical Human Kinetics 6(1): 135-141.

9. Isaac Estevan, Coral Falco, Daniel Jandacka (2011) Mechanical analysis of the roundhouse kick according to the stance position. A pilot study. ISBS-Conference Proceedings Archive.

10. Chadli S, Ababou N, Ababou A (2014) A new instrument for punch analysis in boxing. Procedia Engineering 72: 411-416.

11. Isaac Estevan, Octavio Álvarez, Coral Falcó, Isabel Castillo (2014) Selfefficacy and performance of the roundhouse kick in taekwondo. Revista de artes marciales asiaticas 9(2): 97-105.

12. Estevan I, Alvarez O, Falco C, Molina García J, Castillo I (2011) Impact force and time analysis influenced by execution distance in a roundhouse kick to the head in taekwondo. J Strength Cond Res 25(10): 2851-2856.

13. Isaac Estevan, Coral Falco, Octavio Álvarez, Javier Molina García (2012) Effect of Olympic weight category on performance in the roundhouse kick to the head in taekwondo. J Hum Kinet 31: 37-43.

14. Falco C, Alvarez O, Castillo I, Estevan I, Martos J, et al. (2009) Influence of the distance in a roundhouse kick's execution time and impact force in Taekwondo. J Biomech 42(3): 242-248.

15. Pascal Girodet, Philippe Vaslin, Michel Dabonneville, Patrick Lacouture (2005) Two-dimensional kinematic and dynamic analysis of a karate straight punch. Computer Methods in Biomechanics and Biomedical Engineering 8(S1): 117-118.

16. Tekscan (2016) Sensor: FlexiForce A201.

17. Krzysztof Buśko, Zbigniew Staniak, Szark Eckardt M, Nikolaidis PT, Mazur Różycka J, et al. (2016) Measuring the force of punches and kicks among combat sport athletes using a modified punching bag with an embedded accelerometer. Acta of Bioengineering and Biomechanics 18(1): 47-54.

18. Smith MS, Dyson RJ, Hale T, Janaway L (2000) Development of a boxing dynamometer and its punch force discrimination efficacy. J Sports Sci 18(6): 445-450.

19. Walilko T, Viano DC, Bir CA, (2005) Biomechanics of the head for Olympic boxer punches to the face. Br J Sports Med 39(10): 710-719.

20. Karpiłowski B (1997) Control of the implementation of training tasks using a boxing trainer, [Control of training task by means of boxing simulator]. Trening 2: 159-164.

21. Karpitowski B, Nosarzewski Z, Staniak Z (1994) A versatile boxing simulator. Biology of Sport 11(2): 133-139.

22. Diacu F (2003) On the dynamics of karate. High School Mathematics Magazine Pi in the Sky Volume 6.

23. Bremer AK, Sennwald GR, Favre P, Jacob HA (2006) Moment arms of forearm rotators. Clin Biomech (Bristol, Avon) 21(7): 683-691.

24. Shahbazi M, Sheikh M, Amini A (2008) Kinematic-kinetic comparisons of Tsuki technique in performing from waist and from midway in classic and individual styles. ISBS-Conference Proceedings Archive.

25. Wąsik J, da Silva Santos JF, Franchini E (2013) Movement structure and kinetics of the traditional straight punch: Measurements in taekwon-do athletes. Ido Movement for Culture 13(1): 42-47. 
ISSN: 2574-1241

DOI: 10.26717.BJSTR.2019.14.002550

Nasiriavanaki M. Biomed J Sci \& Tech Res

(C) (i) This work is licensed under Creative

Submission Link: https://biomedres.us/submit-manuscript.php

Assets of Publishing with us
BIOMEDICAL $\quad \begin{aligned} & \text { Global archiving of articles } \\ & \text { - Immediate, unrestricted online access } \\ & \text { - Rigorous Peer Review Process }\end{aligned}$

